\title{
Self reported snoring and daytime sleepiness in men aged 35-65 years
}

\author{
J R Stradling, J H Crosby, C D Payne
}

\begin{abstract}
Background It has been suggested that snoring alone, without conventional sleep apnoea or hypopnoea, may disrupt sleep and produce substantial daytime hypersomnolence. This study addresses this potential relationship.
\end{abstract}

Method Eight hundred and fifty men, aged 35-65 years, drawn from one general practice were visited at home and asked a range of questions potentially related to sleepiness, snoring, and sleep apnoea; these included inquiries about alcohol and cigarette consumption, nasal stuffiness, shift work, hypnotic or other drug use, and medical diagnoses. In addition, measurements of height, weight, and overnight arterial oxygen saturation were made. The relation between snoring and sleepiness, with allowance made for potentially confounding variables, including sleep apnoea, was assessed by multiple logistic regression.

Results Positive answers to all questions about sleepiness were correlated significantly with self reported snoring. After potentially confounding variables and any sleep apnoea had been controlled for, positive answers to four questions about inappropriate drowsiness or sleepiness were independently related to snoring. For example, the odds ratio of admitting to "having almost had two or more car accidents while driving due to sleepiness" was 5.8 (95\% confidence intervals: $2 \cdot 7-12 \cdot 5$ ) in an "often" snorer.

Conclusion Although epidemiological associations such as this do not prove a causal relation, the study suggests that snoring (without classical sleep apnoea) may sometimes reduce sleep quality sufficiently to produce substantial daytime drowsiness.

Osler Chest Unit, Churchill Hospital, Oxford OX3 $7 \mathrm{~L}$

J R Stradling

J H Crosby

Oxford University

Department of Social

Studies, Oxford OX1

2RI

C D Payne

Reprint requests to:

Dr Stradling

Accepted 22 August 1991

In a community survey of sleep apnoea we collected data on self reported snoring, sleepiness, and other symptoms and overnight recordings of arterial oxygen saturation $\left(\mathrm{SaO}_{2}\right)$ by home oximetry from 900 men aged 35-65 tice. ${ }^{1}$ The prevalence of both daytime sleepiness and snoring in this study was considerable. Although answers to three of the questions on sleepiness were correlated to some extent with the number of hypoxaemic dip- ping episodes ( $\mathrm{p}<0.01$ ), answers to the other questions on sleepiness were not. Because snoring alone (in the absence of apnoea, hypopnoea, or hypoxaemia) is now thought to be possibly capable of causing daytime symptoms of sleepiness by disturbing sleep, ${ }^{2}$ we have analysed our data from the 900 men to investigate this hypothesis.

Unfortunately snoring is a complex marker of many other medical problems that might themselves relate to daytime sleepiness, ${ }^{13-6}$ (for example, obesity, nasal blockage from rhinitis, asthma, cigarette consumption, alcohol consumption, and consumption of sedative or other medical drugs). We therefore considered it important in this study to try to allow for as many of these confounding variables as possible before arguing that sleepiness may be related independently to snoring. For example, it was failure to allow for the confounding affect of obesity that led to erroneous assertions that snoring alone might produce diurnal hypertension, when both are probably due to the common precipitant obesity, or, perhaps more correctly, upper body obesity. ${ }^{6-8}$

\section{Methods}

\section{SUBJECTS}

A group general practice of four partners serving the town of Wheatley, five miles east of Oxford, allowed us to use their age and sex register to identify all the men aged 35-65 years (1001) who could be contacted by telephone or letter. Subjects were seen over four years. They were selected sequentially according to their year of birth, but the year-of-birth order was randomised. Each subject received a letter of explanation, signed by his general practitioner, followed by a telephone call or letter from one of us. Nine hundred $(90 \%)$ agreed to be studied and no subject was excluded from the study for any reason other than refusal to participate. Complete data for this analysis of snoring and sleepiness were available on 855 subjects, non-drivers accounting for most of the incomplete sets of data years, drawn from one group general prac-

\section{TECHNIQUES}

A questionnaire was administered to all subjects by the same individual throughout the study. This inquired about a range of factors thought to be potentially important in snoring, sleepiness, and sleep apnoea. Where possible, the questions demanded a choice between "never", "rarely", "sometimes", and "often." We asked several different questions about sleepiness (table), which in clinical 
Overall distributions of answers (in percentages) to the individual questions about sleepiness in 855 men: results divided according to whether the subjects were "often" snorers (16.9\%) or answered "never", "rarely" or "sometimes" (amalgamated, $83 \cdot 1 \%$ )

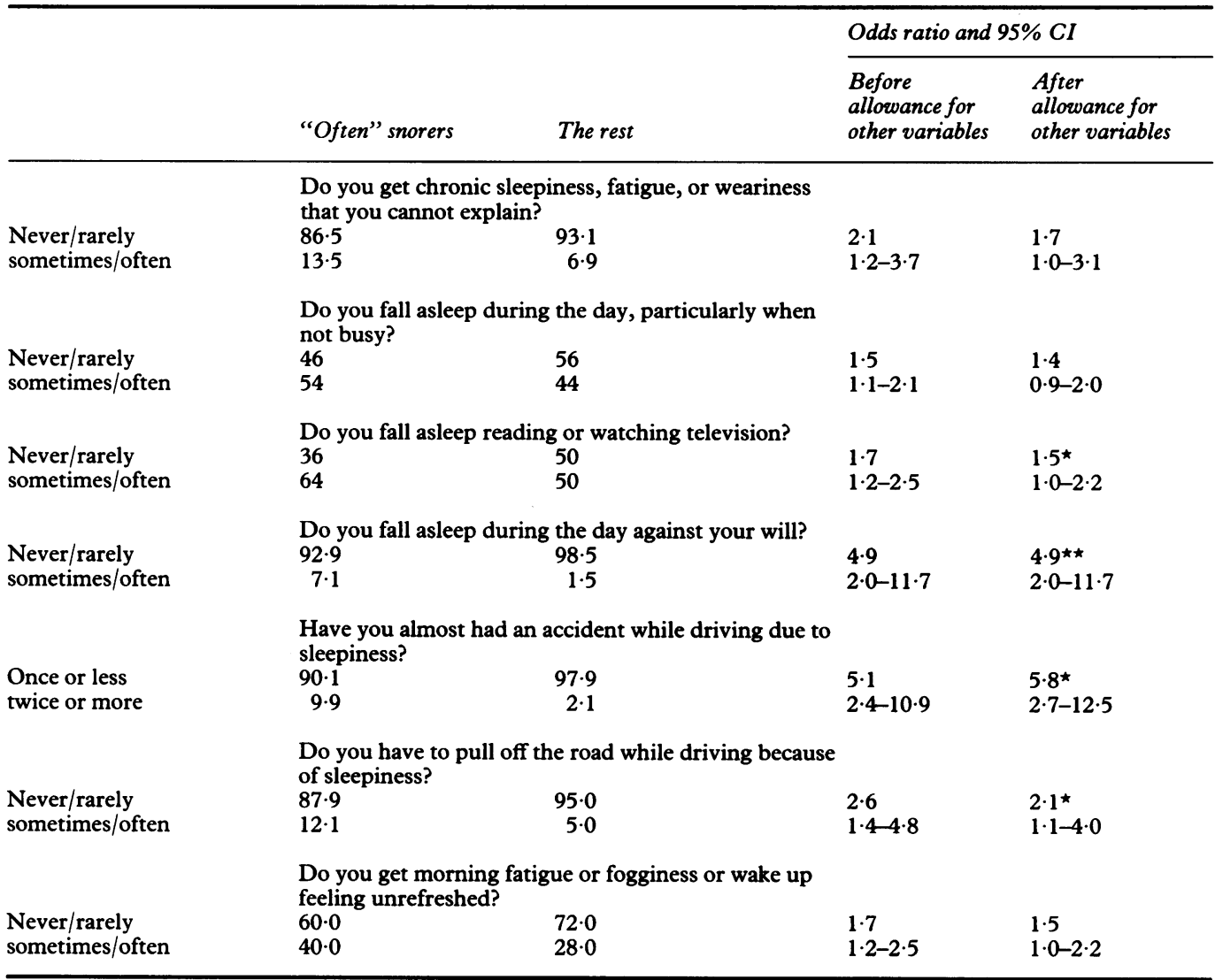

^Remains significant after allowance for other variables.

$\star \star$ No other variables were significantly related and therefore odds ratio remains unchanged.

practice are usually answered positively by patients with obstructive sleep apnoea. In addition, we asked about snoring, use of hypnotics or sedatives, and nasal stuffiness. Other questions included "Have you any other medical problems" (yes or no), "Are you taking any medications" (yes or no), level of alcohol consumption (categories 1-4, <10, $10-39,40-69, \geqslant 70 \mathrm{~g} /$ day), level of cigarette consumption (categories 1-4: nil, 1-5, 6-15, $>15$ (day), and whether the subject did shift work (yes or no). The usual sleep time was also recorded, and whether the subject's wife was present at the interview.

We measured height and weight to provide the body mass index $\left(\mathrm{kg} / \mathrm{m}^{2}\right)$. Overnight oxygen saturation was measured in the subject's own home (Ohmeda 3700). The stored overnight $\left(\mathrm{SaO}_{2}\right)$ data (eight hours) from the oximeter were transferred into a computer the following day and analysed for number of drops in $\mathrm{SaO}_{2}$ of more than $4 \%$ with an algorithm described elsewhere. ${ }^{7}$ This provided a measure of severity of sleep apnoea.

\section{STATISTICS}

All data were entered into the Oxford University VAX computer. The data were then explored and analysed by means of the general linear interactive modelling (GLIM) statistical package, ${ }^{9}$ which allows mixing of contin- uous and discontinuous predictors. The four point answers to each question on sleepiness were collapsed to a two point answer-never/ rarely and sometimes/often (table). Single logistic regression was then used to establish the effect of "often" snoring on the odds ratio (and $95 \%$ confidence limits) for each of the questions on sleepiness (in relation to "never," "rarely", or "sometimes" a snorer). Then for each question on sleepiness all the other potentially important variables were entered first into a logistic regression model, snoring being entered last. The odds ratio for the effect of "often" snoring on each question about sleepiness was thus established, both with and without control for all the potentially confounding variables.

\section{Results}

In the single logistic regression analysis positive answers to all the questions about sleepiness (table) were significantly associated with self reported "often" snoring, with odds ratios of up to $5 \cdot 1$ for the question "Have you almost had an accident while driving due to sleepiness?" Some of the other factors were also associated with positive answers to the questions on sleepiness, as would be expected. For example, the use of hypnotics produced a highly significant odds ratio for the question "Do you get morning fatigue or fogginess or 
wake up feeling unrefreshed?" $(8 \cdot 8,95 \%$ confidence interval $2 \cdot 8-28 \cdot 2$ ). None of the factors significantly affected answers to all the questions on sleepiness as snoring did. The question showing significant effects from most factors was "Do you get chronic sleepiness, fatigue, or weariness that you cannot explain?" As might be expected, the presence of other illness $(2.0$, CI 1.2-3.4) and use of medical drugs $(1 \cdot 8, \mathrm{CI} 1 \cdot 1-3 \cdot 1)$ were significantly related to a positive answer to this question, as was nasal stuffiness (3.1, CI 1.5-6.6). Age was significantly related to falling asleep while reading or watching television $(p<0.0001)$. High alcohol consumption was significantly related only to a positive answer to the question "Do you have to pull off the road while driving because of sleepiness" (5.4 CI 1·7-17.3).

In the multiple logistic regression analysis positive answers to four of the seven questions about sleepiness were still significantly related to snoring after all the other significant factors in this study had been controlled for (table). Our measure of sleep apnoea severity (hypoxaemic dipping rate overnight) was significantly related to answers to three of the questions on sleepiness $(p<0.01)$, but it was never more important than snoring as a predictor of sleepiness and never reduced the significance of this.

A reanalysis of the data using only subjects where the wife was present at the interview (52\% of interviews) did not significantly alter the above results, even though the prevalence of "often" snoring in this group was $23 \%$ compared with only $10 \%$ in the group without wives present at the interview $(16.9 \%$ prevalence overall).

\section{Discussion}

This study has shown that complaints of daytime sleepiness are associated with self reported snoring in middle aged men selected at random. This association remained significant after many potentially confounding variables had been controlled for, particularly when the questions related to overwhelming sleepiness and sleepiness while driving. Snoring was a better predictor of sleepiness than our direct measure of sleep apnoea or sleep hypopnoea severity-the number of hypoxaemic dips (falls in $\mathrm{SaO}_{2}$ of more than $4 \%$ ) during sleep. This suggests that snoring, in the absence of classical sleep apnoea, may cause sleepiness. Perhaps, however, this association between self reported snoring and sleepiness is due to another, unmeasured, confounding third variable or variables. In addition, we were not able to check whether the association was produced by a personality that would freely admit to various problems; but, if this were so, it would not explain why snoring, unlike any other variable, was consistently associated with positive answers to all the questions on sleepiness.

Alternatively, perhaps sleepiness (from other reasons) causes snoring rather than the reverse, as excessive tiredness may cause greater snoring. ${ }^{111}$ We did not, however, find an association between reported sleep time and either snoring or sleepiness. This indirectly suggests that inadequate, shorter sleep times are not the cause of the association.

The data were collected by an administered questionnaire with a $90 \%$ response rate and this is more likely to be reliable than postal questionnaires. Sampling bias seems unlikely to have produced this result. The actual history of snoring may be relatively unreliable because we and others have observed discrepancies between the history and objective measures of snoring. If only objective snoring were considered possibly the association with sleepiness might be stronger.

The different questions we used to assess sleepiness covered different aspects of this symptom. Feeling unrefreshed in the morning is different from falling asleep against one's will, or while driving, and is different again from being chronically fatigued or weary. This is presumably why use of hypnotics significantly and independently influenced morning symptoms but not daytime ones. The question about near car accidents was influenced by age, perhaps because driving experience and the opportunity for near accidents, or alternatively perception of impaired performance generally, increases with age. Nasal stuffiness, rather unexpectedly, was related to positive answers to some questions on sleepiness, not only as a partial covariant with snoring but also independently. This may be explained by the observations of Lavie et al, ${ }^{12}$ who obstructed (both partially and completely) the nasal passages of normal young adults during sleep and showed that, as well as causing a small increase in the number of apnoeic events, this produced nonapnoeic breathing irregularities that provoked recurrent short arousals.

This association between snoring and sleepiness raises the possibility that snoring may disturb sleep without the characteristic hypoxaemic events that go with apnoea or hypopnoea. Although not all cases of classically defined apnoea or hypopnoea always have falls in $\mathrm{SaO}_{2}$ of more than $4 \%$, the correlation between the rate of apnoeic events (the apnoea index) and the desaturation index is extremely close. ${ }^{1314}$ Thus failure by us to diagnose all the cases of conventional sleep apnoea, because of reliance on hypoxaemic events, is very unlikely to be the reason why snoring is a better predictor or sleepiness than the overnight desaturation index.

Block's group, ${ }^{15}{ }^{16}$ studying men selected by local newspaper advertising, suggested that loud snoring, without much sleep apnoea, might produce daytime sleepiness and neuropsychological deficits. Telakivi et al compared snorers and non-snorers and found a weak correlation, but a significant number of the snorers had periods of sleep apnoea ${ }^{17} \mathrm{Schmidt-}$ Nowara $e t a l^{6}$ showed in men and women aged over 18 years that snoring was correlated with positive answers to questions about sleepiness. This study used multiple logistic regression to show that snoring and blood pressure were only secondarily related to each other via obesity, but this technique was not used to explore the possibility that snoring and sleepiness were 
only secondarily related via another variable, and the link was assumed to be via occult classical sleep apnoea. Billiard et al studied young men and showed by questionnaire alone that sleepiness and snoring were independently correlated, but hypnotics were the only relevant covariable included in the analysis. ${ }^{18}$

Most units investigating patients with hypersomnolence and snoring find that only a proportion have classical sleep apnoea. Comparison of the groups with and without sleep apnoea shows the non-apnoeic group to be less obese and only a little less sleepy. ${ }^{15}{ }^{19}$ In the past we and others have assumed that patients in the non-apnoeic group do not have a sleep related breathing disorder and must have other reasons for their sleepiness. Possibly this approach is wrong and successful treatment of the snoring would relieve the sleepiness. If treatment does prove to be helpful, then presumably in some individuals snoring produces a subtle disruption of the continuity of sleep, perhaps not evident from conventional sleep staging. ${ }^{20} \mathrm{~A}$ preliminary report from Stanford suggests this may be true. ${ }^{2}$

Gleeson et al have found in normal subjects that arousal due to either hypoxia, hypercapnia, or resistive loading correlates better with the extent of pleural pressure swings than with any blood gas disturbances. ${ }^{21}$ Arousal could be consistently produced by pressure swings as small as $-13 \mathrm{~cm} \mathrm{H} \mathrm{H}_{2} \mathrm{O}$, much less than is found in some snorers. ${ }^{22}$ An alternative hypothesis is that the greater effort required to generate these recurrent, bigger negative intrathoracic pressure swings found in loud snorers somehow produces daytime symptoms of sleepiness or fatigue in the absence of disruption of sleep architecture, as has been suggested in children. ${ }^{23}$

If the hypothesis that snoring causes daytime sleepiness is correct, then it represents a considerable problem that could to some extent be soluble. In our study population $17 \%$ claimed to be "often" snorers. Had they not been snorers only $2 \cdot 1 \%$ would have been expected to admit to having almost had more than one accident while driving due to sleepiness, whereas $9.9 \%$ actually did so. This means that around $7 \cdot 8 \%$ of $17 \%$ or overall $1.3 \%$ of men aged 35-65 years $(13 / 1000)$ are perhaps at risk of having potentially fatal car accidents through snoring induced hypersomnolence. Snoring is sometimes due to reversible causes such as obesity, drinking alcohol, nasal blockage, or smoking ${ }^{13-6}$ and may thus be treatable.

Clearly many aspects of this hypothesis, that snoring may sometimes lead to substantial daytime somnolence, need further investigation before a cause and effect association can be inferred. This work was supported by the Wellcome Trust. We thank the general practitioners in Wheatley-Drs $R$ Flury, $P$ Isaac, which this study would not have been possible.

1 Stradling JR, Crosby J. Predictors and prevalence of obstructive sleep apnoea and snoring in 1001 middle aged men. Thorax 1991;46:85-90.

2 Guilleminault C, Stoohs R, Duncan S. Snoring. Daytime sleepiness in regular heavy snorers. Chest 1991;99:40-8.

3 Bloom JW, Kaltenborn WT, Quan SF. Risk factors in a general population for snoring. Importance of cigarette smoking and obesity. Chest 1988;93:678-83.

4 Lugaresi E, Cirignotta F, Coccagna G, Piana C. Some epidemiological data on snoring and cardiocirculatory disturbances. Sleep 1980;3:221-4.

5 Norton PG, Dunn EV. Snoring as a risk factor for disease. BMJ 1985;291:630-2.

6 Schmidt-Nowara WW, Coultas DB, Wiggins C, Skipper $B E$, Samet JM. Snoring in a Hispanic-American population. Risk factors and association with hypertension and other morbidity. Arch Intern Med 1990;150:597-601.

7 Stradling JR, Crosby JH. Relation between systemic hypertension and sleep hypoxaemia or snoring: analysis in 748 men drawn from general practice. $B M J 1990 ; 300: 75-8$.

8 Hoffstein V, Rubinstein I, Mateika S, Slutsky AS. Determinants of blood pressure in snorers. Lancet 1988;ii:992-4.

9 Payne C, ed. The GLIM system release 3.77 manual. Oxford: Royal Statistical Society and Numerical Algorithms Group, 1985.

10 Lugaresi E, Cirignotta F, Montagna P. Snoring: pathogenic, clinical and therapeutic aspects. In: Kryger MH, Roth T, Dement WC, eds. Principles and practice of sleep medicine. Philadelphia: Saunders, 1989:494-500.

11 Leiter JC, Knuth SL, Bartlett D. The effect of sleep deprivation on activity of the genioglossus muscle. Am Rev Respir Dis 1985;132:1242-5.

12 Lavie P, Fischel N, Zomer J, Eliaschar I. The effects of partial and complete mechanical occlusion of the nasal passages on sleep structure and breathing in sleep. Acta passages on sleep structure

13 Farney RJ, Walker LE, Jensen RL, Walker JM. Ear oximetry to detect apnea and differentiate rapid-eyemovement (REM) and non-REM (NREM) sleep. Chest 1986;89:533-9.

14 George CF, Millar TW, Kryger MH. Identification and quantification of apneas by computer-based analysis of oxygen. Am Rev Respir Dis 1988;137:1238-40.

15 Berry DTR, Webbe WB, Block AJ, Switzer DA. Sleepdisordered breathing and its concomitants in a subclinical population. Sleep 1986;9:478-83.

16 Block AJ, Berry D, Webb W. Nocturnal hypoxemia and neuropsychological deficits in men who snore. Annu Rev Med 1986;69:405-8.

17 Telakivi T, Kajaste S, Partinen M, Koskenvuo M, Salmi T, Kaprio J. Cognitive function in middle-aged snorers and controls: role of excessive daytime somnolence and sleeprelated hypoxic events. Sleep 1988;11:454-62.

18 Billiard M, Alperovitch A, Perot C, Jammes A. Excessive daytime somnolence in young men: prevalence and contributing factors. Sleep 1987;10:297-305.

19 Crocker BD, Olson LG, Saunders NA, Hensley MJ, McKeon JL, Allen KM, et al. Estimation of the probability of disturbed breathing during sleep before a sleep study. Am Rev Respir Dis 1990;142:14-8.

20 Rechtschaffen A, Kales A. A manual of standardised terminology, techniques and scoring system for sleep stages of human subjects. Washington DC: Public Health Service, 1968 (National Institutes of Health publication No 204.)

21 Gleeson K, Zwillich CW, White DP. The influence of increasing ventilatory effort on arousal from sleep. Am Rev Respir Dis 1990;142:295-300.

22 Lugaresi E, Coccagna G, Farneti P, Mantovani M, Cirignotta F. Snoring. Electroencephalog Clin Neurophysiol 1975;39:59-64.

23 Guilleminault $C$, Winkle R, Korobkin R, Simmons B. Children and nocturnal snoring: evaluation of the effects of sleep related respiratory resistive load and daytime functioning. Eur J Pediatr 1982;139:165-71. 